\title{
An event-related fMRI study of the neurobehavioral impact of sleep deprivation on performance of a delayed-match-to-sample task
}

\author{
Christian Habeck $^{\mathrm{a}}$, Brian C. Rakitin ${ }^{\mathrm{a}, \mathrm{b}}$, James Moeller ${ }^{\mathrm{a}, \mathrm{c}}$, Nikolaos Scarmeas ${ }^{\mathrm{a}, \mathrm{b}, \mathrm{d}}$, \\ Eric Zarahn ${ }^{\mathrm{a}, \mathrm{c}}$, Truman Brown ${ }^{\mathrm{e}}$, Yaakov Stern ${ }^{\mathrm{a}, \mathrm{b}, \mathrm{c}, \mathrm{f}, *}$ \\ ${ }^{a}$ Cognitive Neuroscience Division of the Taub Institute for Research in Alzheimer's disease and the Aging Brain, 630 West 168th Street, PH-18, \\ New York, NY 10032, USA \\ ${ }^{\mathrm{b}}$ Department of Neurology, College of Physicians and Surgeons of Columbia University, 630 West 168th Street, New York, NY 10032, USA \\ ${ }^{\mathrm{c}}$ Department of Psychiatry, College of Physicians and Surgeons of Columbia University, 630 West 168th Street, New York, NY 10032, USA \\ ${ }^{\mathrm{d}}$ Department of Medicine, College of Physicians and Surgeons of Columbia University, 630 West 168th Street, New York, NY 10032, USA \\ ${ }^{\mathrm{e}}$ Department of Biomedical Engineering, College of Physicians and Surgeons of Columbia University, 630 West 168th Street, New York, NY 10032, USA

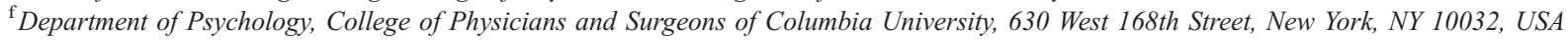

Accepted 30 October 2003

\begin{abstract}
Eighteen subjects (ages 18-35) underwent event-related functional magnetic resonance imaging (efMRI) while performing a delayedmatch-to-sample (DMS) task before and immediately after $48 \mathrm{~h}$ of sustained wakefulness. The DMS trial events were: a 3-s study period of either a one-, three-, or six-letter visual array; a 7-s retention interval; and a 3-s probe period, where a button press indicated whether the probe letter was in the study array. Ordinal Trend Canonical Variates Analysis (OrT CVA) was applied to the data from the probe period for trials with six-letter study lists prior to and immediately following sleep deprivation to find an activation pattern whose expression decreased with sleep deprivation in as many subjects as possible, while being present in both conditions. The first principal component of the OrT analysis identified a covariance pattern whose expression decreased as a function of sleep deprivation in 17 of 18 subjects $(p<0.001)$. While overall expression of the pattern showed a systematic decrease with sleep deprivation, the brain regions that make up the pattern show covarying increases and decreases in activation. Regions that decreased their activation were noted in the parietal (BA 7 and 40 ), temporal (BA 37, 38 and 39) and occipital (BA 18 and 19) lobes; regions that increased their activation were noted in the cerebellum, basal ganglia, thalamus and the anterior cingulate gyrus (BA 32). The reduction in pattern expression with sleep deprivation for each subject was related to the change in performance on the DMS task. Subject decreases in pattern expression were correlated with reductions in recognition accuracy $(p<0.05)$, increased intra-individual variability in reaction time $(p<0.005)$ and increased lapsing $(p<0.005)$.
\end{abstract}

(C) 2003 Elsevier B.V. All rights reserved.

Theme: Neural basis of behavior

Topic: Cognition

Keywords: Covariance analysis; Functional imaging; Neural networks; Non-verbal recognition memory; Sleep deprivation

\section{Introduction}

The brain consists of a set of functionally distinct regions, yet, these regions are also interconnected to form functional networks. While the profound effects of sleep deprivation on

* Corresponding author. Cognitive Neuroscience Division of the Taub Institute for Research in Alzheimer's Disease and the Aging Brain, 630 West 168th Street, P\&S Box 16, New York, NY 10032, USA. Tel.: +1-212305-9194; fax: +1-212-305-2426.

E-mail address: ys11@columbia.edu (Y. Stern). behavior has been a topic of study for over a century, only recently have technological innovations allowed investigation of the changes to the human brain before and during sleep deprivation that may underlie those behavioral changes. Among those studies that have approached this critical issue, the emphasis has been on identifying individual brain areas affected by sleep deprivation. In the study reported here, we seek to identify changes to pre-existing functional networks that support basic psychomotor and short-term memory function. With this goal in mind, we have chosen to examine changes to the MR signal before and 
during profound sleep deprivation obtained while subjects perform a speeded short-term memory task descended from the Sternberg paradigm. In addition to basic visual, motor and short-term memory function, this paradigm also provides an internal manipulation of difficulty useful in addressing the specificity of the observed brain networks.

Sleep deprivation significantly impacts human functioning [37], negatively affecting levels of alertness and cognitive performance $[25,45]$. It has been demonstrated that sleep-deprived individuals experience increased reaction times and reduced vigilance relative to those with normal sleeping patterns $[2,3,26,28,30,45]$.

The behavioral effects of sleep deprivation in speeded response tasks can be integrated under a general umbrella of phenomena observed for sleep deprivation which was reviewed comprehensively by Dinges and Kribbs [13]. This framework involves five key concepts that represent independent aspects: (1) cognitive slowing, (2) lapsing, (3) memory effects, (4) time-on-task effects and (5) optimumresponse shifts. It appears that these five phenomena can account for many of the performance decrements to varying degrees, depending on the characteristics of the cognitive task under study. This contrasts with earlier, neuropsychologically inspired, theories that posit sleep deprivation effects on specific higher cognitive function similar to the effects of lesions [13].

In addition to behavioral studies, an increasing number of neuroimaging studies have recently investigated the effects of sleep deprivation. Changes in cerebral blood flow may relate to the cognitive deficits observed during sleep deprivation $[16,27]$. Neuroimaging studies, using positron emission topography (PET) [45,47] or functional magnetic resonance imaging (fMRI) [16], have shown that changes in cerebral activation occur as a function of sleep deprivation, and that these changes are associated with changes in cognitive performance. Significant decreases in overall glucose metabolism following $32 \mathrm{~h}$ of sleep deprivation were first reported in 1991 [47]. Since then, significant decreases in overall glucose metabolism following $24 \mathrm{~h}$ of sleep deprivation [45] were also observed during performance of a serial subtraction task and shown to correlate with declines in performance. Decreases in regional glucose activity have been observed primarily in the thalamus [45,47], temporal [47], prefrontal and parietal cortices [45].

Using fMRI on the other hand, Drummond et al. [16] reported an increase in cerebral activation of the bilateral prefrontal cortex (LPFC) and parietal lobes following $35 \mathrm{~h}$ of sleep deprivation. Further, less impairment on a divided attention task, involving both a verbal learning and an arithmetic task, was associated with greater activation of the left inferior parietal/superior temporal gyri and the right inferior parietal gyri. On a verbal learning task, greater activation of the bilateral parietal lobe was associated with better task performance [15].

All of the imaging studies mentioned before used univariate region-by-region analyses, paying tribute to the well- documented functional segregation in the brain and implicitly adopting the lesion model of sleep deprivation. Instead, we wanted to perform a complementary analysis and capture the well-characterized global deficits of sleep deprivation mentioned in the previous paragraph, reasoning that they might result in changes in activation in a network of more widely distributed brain regions, in addition to focal deficits. For identifying a set of brain regions whose covarying changes in activation could account for the observed performance deficits, we had to use a multivariate analysis technique, rather than analyzing the brain activation on a region-by-region basis.

Event-related functional magnetic resonance imaging (efMRI) data were used in order to establish neural correlates of the observed performance decrements. We adopted a delayed-match-to-sample (DMS) task that was a variant of the Sternberg memory-scanning task for our study of sleep deprivation. This short-term memory task offers a manipulation of difficulty in terms of memory load and allows precise chronometric information on subjects' performance. Our version of the task contains an encoding phase (stimulus), rehearsal phase (retention) and a retrieval phase (probe). This short-term memory task has been studied extensively inside and outside the scanner (e.g., Refs. $[39,41,43,44])$, is easy to administer and taps a set of cognitive component processes (pertaining to visual encoding, rehearsal, memory scanning, binary decision, response selection and motor output). We set out to investigate which ones of these component processes might stay relatively intact, and which ones might be affected adversely by sleep deprivation. Of particular interest to us was the probe phase of the task, which involved memory scanning, binary decision, response selection and motor output processes.

Furthermore, there is a practical reason why the probe phase is best suited for the analysis of the effects of sleep deprivation. Sleep deprivation causes subjects to lapse and miss the deadline for responses during the probe phase. Event-related fMRI data from such non-response trials are excluded for further analysis. The probe period is the task component where responses and non-responses have the most predictive power and are therefore best suitable for ascertaining whether subjects actually performed the task (which becomes important after $48 \mathrm{~h}$ of sustained wakefulness). During the stimulus and the retention phase on the other hand, there is a greater possibility of misclassification: If subjects paid attention during the stimulus phase, rehearsed the items during the retention phase, but failed to make a response in the probe phase, the trial would be lostactivation that should have been included from the point of view of the stimulus and retention phase would be thrown out. This is also why we were primarily interested in the extent to which changes in neural activation during the probe phase resulted from sleep deprivation and could be related to worsening task performance.

DMS tasks have been studied extensively in recent years, and the advances in event-related imaging techniques facil- 
itated the separation of the different cognitive processes involved in task performance into different temporal components $[12,39,42]$. Of particular interest is the role of the LPFC and its relation to the number of items that have to be retained in the task (memory load). A recent event-related study [42] of a DMS task with serial presentation of one to eight letters found decreasing activation in the ventral portion of the LPFC (BA 44,45,47) with increasing memory load during encoding, whereas the dorsal portion of the $\operatorname{LPFC}(\mathrm{BA} 9,46)$ displays increasing activation with increasing memory load during encoding. Consistent with this finding is the different role of ventral and dorsal portions of the LPFC: Dorsal LPFC becomes involved once the quantity of the to-be-remembered information exceeds the working-memory capacity limit of "4 \pm 1 items" [10], necessitating executive "chunking" processes to encode information successfully. Presumably, the ventral portion of the LPFC, which is geared towards maintenance and rehearsal processes at below-capacity levels, would then decrease its involvement. For the probe period, a recent study [42] found load-dependent modulation in the activation of both dorsal and ventral LPFC. This loaddependent activation was also beneficial for task-performance, i.e., the more subjects activated dorsal LPFC during the probe period in a load-dependent manner, the more accurately they classified the probe letter. On the other hand, overall activation (regardless of memory load) in the LPFC was lower for subjects that performed faster and more accurately.

Because of the pivotal role of the prefrontal cortex in short-term memory tasks, it has also figured prominently in the extant literature on sleep-deprivation with at times contradictory findings: Of the studies mentioned earlier, two $[15,16]$ reported increased PFC activation as a result of sleep loss, whereas two other studies $[14,45]$ reported decreased PFC activation. Studies [14-16] used $35 \mathrm{~h}$ of sleep deprivation, while [45] used $24 \mathrm{~h}$. The different response of the prefrontal cortex to sleep deprivation therefore could arise either from the difference in the cognitive tasks used and the additional attentional demands exerted by the dual-task situation, or by the difference in the degree of sleep deprivation. Another possible confound might be presented by circadian effects as the amount of sleep deprivation incurred by subjects in the two types of studies was not an integer multiple of $24 \mathrm{~h}$ and testing occurred at different times of day $[2,3,35]$. This makes comparison across studies difficult, even when the studies individually used adequate controls.

On the basis of these findings it is difficult to cast a hypothesis of (1) how the LPFC responds to sleep deprivation and (2) whether this response is beneficial or detrimental to performance in our DMS task. We therefore chose to expand on these results with a multivariate approach that pays attention to individual differences in regional MR signal covariance. Multivariate approaches can disambiguate different influences (in our case: sleep deprivation and memory load) that might be acting simultaneously on a brain region by utilizing covariation of activation with that of other brain regions.

In addition, our method demands a consistent sleepdeprivation-induced change in brain activation in a distributed set of brain regions for as many subject as possible, rather than just allowing a mean change that could be caused by overly influential subjects. We specifically searched for an activation pattern common to both PRE and POST conditions, with a decrease in expression from PRE to POST for as many subjects as possible.

\section{Methods}

Eighteen healthy subjects, between the ages of 20 and 35 years (age $=26.3 \pm 4.9$ years), participated in an efMRI paradigm of a DMS task. The initial scan occurred at 9 AM (PRE), and the follow-up scan occurred at the same time $48 \mathrm{~h}$ later (POST) to eliminate confounding circadian effects, yielding $48 \mathrm{~h}$ of prolonged wakefulness. All subjects were right-handed and carefully screened to ensure that they had no history of medical, psychiatric, neurological or sleep disorder. Subjects maintained a sleep log for 2 weeks prior to study; the average amount of sleep per subject and per day was $8.00 \mathrm{~h}$. Mean within-subjects variability across the 14 days prior to the experiment gave a mean standard deviation of $0.95 \mathrm{~h}$. One subject slept $6 \mathrm{~h}$ on both nights preceding the experiment, while the rest slept more than 6 $\mathrm{h}$ on both these nights. We examined whether the withinsubjects means and STD correlated with any of the performance variables or neural activation to be discussed below, and obtained no significant findings.

Subjects were instructed to stop drinking caffeine 24 $\mathrm{h}$ prior to study participation and for the duration of the study. All subjects passed substance abuse screening tests. Subjects were supervised at all times, and polysomnographic monitoring confirmed that they remained awake during the sleep deprivation period.

Fourteen control subjects (age 23.93 \pm 1.14 ) underwent the same protocol without being sleep deprived. Apart from one exception, the control subjects also maintained a sleep $\log$ for 2 weeks prior to the study. The average amount of sleep per subject was $7.96 \mathrm{~h}$, and the mean within-subjects standard deviation of $1.08 \mathrm{~h}$. Of the 13 subjects who handed in their sleep logs, 12 had at least $6 \mathrm{~h}$ of sleep on both nights prior to the experiment.

Informed consent, as approved by the Internal Review Board of the College of Physicians and Surgeons of Columbia University, was obtained prior to study participation and after the nature and risks of the study were explained. Subjects were paid for their participation in the study.

\subsection{Delayed-match-to-sample task}

The DMS task was a variant of the Sternberg task $[43,44]$. A trial lasted a total $16 \mathrm{~s}$. Subjects were instructed 
to respond as accurately as possible. No feedback about their performance was given. The sequence of trial events was as follows (Fig. 1): First, a fixed 3-s period of blank presentation marked the beginning of trial; then, during the stimulus period of the task, an array of one, three or six capital letters were presented for $3 \mathrm{~s}$ (stimulus phase).

With the offset of the visual stimulus, subjects were instructed to focus on the blank screen and hold the stimulus items in mind for a 7-s maintenance interval (retention phase). Finally, a probe appeared for $3 \mathrm{~s}$ (probe phase), which was a lowercase letter centered in the field of view. In response to the probe, subjects indicated by a button press whether or not the probe matched a letter in study array. (Left index finger indicated "Yes", right index finger indicated "No".)

Each experimental block contained 10 trials for each of three set sizes with five true negative and five true positive probes per set size. Three experimental blocks were run in total, yielding $10 \times 3 \times 3=90$ experimental trials per scanning session. In addition to the fixed 3-s period of a blank screen presentation, which we counted as part of the experimental trial, there were inter-trial intervals (ITI) that consisted of presentation of a blank screen and were used as baseline epochs in the time series analysis of the subject's data. Their length was variable and determined in the following way: Seventy 2 -s increments were available throughout the whole block for 30 inter-trial intervals. It was decided probabilistically whether a 2-s increment of ITI would be inserted prior to the start of the trial, or whether the trial would begin immediately. The probabilities for choosing between additional ITI increments or starting the experimental trial were updated according to a sampling- without-replacement rule: For example, at the start of the experimental block, the probabilities for choosing a 2-s ITI increment versus starting the experimental trial were 70/100 versus 30/100. If a 2-s ITI increment was chosen, these probabilities would be updated to 69/99 versus 30/99 for the decision of adding another ITI increment versus starting the trials. This sampling rule keeps the overall time assigned to ITI constant at $70 \times 2=140 \mathrm{~s}$ per block. With 30 trials of 16 s each, each block therefore lasted for $140+(30 \times 16)=620$ s. There were two breaks of approximately a minute each between blocks 1 and 2 as well as blocks 2 and 3. This makes the overall time subjects spent in the scanner during each session $(3 \times 620)+120=1980 \mathrm{~s}$, or $33 \mathrm{~min}$.

Subjects as well as controls went through a training run of seven blocks on the evening prior to the start of the experiment, the first six of which were administered with feedback. The training session was conducted to eliminate confounding effects from learning through repeating the performance of the DMS task.

\section{2. fMRI acquisition and processing}

Functional images were acquired using a 1.5-T magnetic resonance scanner (Philips). A gradient echo EPI sequence $\left[\mathrm{TE}=50 \mathrm{~ms} ; \mathrm{TR}=3 \mathrm{~s}\right.$; flip angle $\left.=90^{\circ}\right]$ and a standard quadrature head coil was used to acquire T2*-weighted images with an in-plane resolution of $3.124 \times 3.124 \mathrm{~mm}$ $\left(64 \times 64\right.$ matrix; $20 \mathrm{~cm}^{2}$ field of view). Based on $\mathrm{T}_{1}$ "scout" images, 8-mm transaxial slices (15-17) were acquired. Following the fMRI runs, a high (in-plane) resolution $\mathrm{T}_{2}$ image at the same slice locations used in the fMRI run was acquired using a fast spin-echo sequence $[\mathrm{TE}=100$

\section{The Delayed Match-to-Sample Task (Sternberg Variant)}

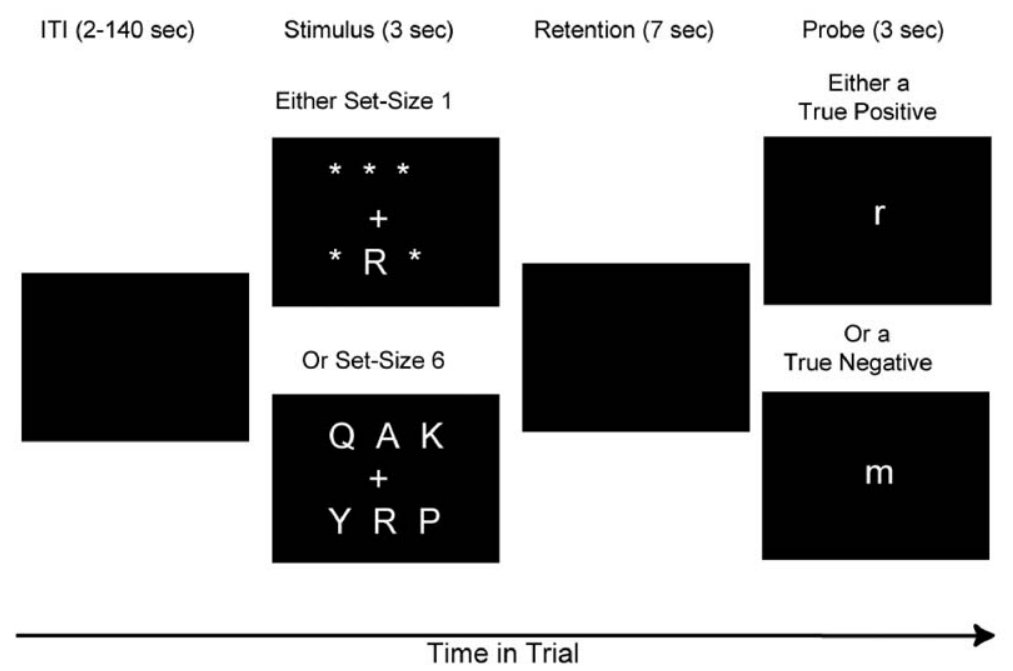

Fig. 1. Schematic sketch of delayed-match-to-sample paradigm of the Sternberg variant. 
ms; TR $=3 \mathrm{~s} ; 256 \times 256$ matrix; $20 \mathrm{~cm}^{2}$ field of view]. Task administration and data collection will be controlled by a computer running appropriate software (Psyscope 1.1), and electronically synchronized with the MR scanner. Task stimuli are back-projected onto a screen located at the foot of the MRI bed using an LCD projector. Subjects view the screen via a mirror system located in the head coil. Task responses are made on a LUMItouch response system and behavioral response data is recorded on the task computer.

All image processing and analysis was done using the SPM99 program (Wellcome Department of Cognitive Neurology) and other code written in Matlab 5.3 (Mathworks, Natick, MA). fMRI time series were corrected for order of slice acquisition. All functional volumes in a given subject were realigned to the first volume from the first run of each study. The $T_{2}$ anatomical image was then co-registered to the first functional volume, using the mutual information co-registration algorithm implemented in SPM99. This coregistered structural image was then used in determining non-linear spatial normalization $(7 \times 8 \times 7$ nonlinear basis functions) parameters for a transformation into a Talairach standard space defined by the Montreal Neurological Institute template brain applied with SPM99. These normalization parameters were then applied to the functional data (using SINC-interpolation to reslice the images to $2 \times 2 \times 2 \mathrm{~mm})$.

\subsection{Data analysis}

The fMRI responses to the three separate temporal components of the task, in each experimental condition and in each block, were fit to separate sets of predictor variables [48]. The predictor variables that were ultimately used in the first-level model estimation were obtained in the following way: a constant intercept (zeroth-order discrete cosine set) was chosen for the stimulus and probe phases, whereas a zeroth- to second-order discrete cosine set was chosen for the retention phase. For one block this results in five predictor variables (one for stimulus, three for retention, one for probe) per set size (1, 3 and 6$)$ per probe type (positive or negative). An additional intercept term is provided for the effect of block, bringing the total number of predictor variables per block to $(5 \times 3 \times 2)+1=31$. Predictor variables had a non-zero value at every point in the time series where a particular condition was met, and a zero value at every other point. For example, one predictor had a value of one during all stimulus phases of set size one, with a positive probe, during the first block.

The set was convolved with a canonical hemodynamic response waveform (a sum of two gamma functions, as specified in the SPM99 program [19]) whose beginnings were marked by the appropriate onset vector for each epoch, set size and probe type. The resulting time series vectors were used in the design matrix for the within-subjects model estimation. The final matrix had $3 \times 3$ block-diagonal form. The number of rows was the total number of volumes denoting the complete fMRI time series across the scanning session. The number of columns was $3 \times 31=93 ; 31$ design vectors for each experimental block as explained above.

The band-pass filtered (low pass by a Gaussian with a FWHM of $4 \mathrm{~s}$ and a high pass cutoff of $14.5 \mathrm{mHz}$ ) fMRI time series at each voxel were regressed onto these predictor variables. A first-order autoregressive autocorrelation model was fit to the residuals to make statistical inference more robust to the intrinsic temporal autocorrelation structure [20].

At every voxel in the image, contrasts assessed the amplitudes (normalized regression coefficients) of the components of the event-related responses that matched the canonical hemodynamic response waveform for the whole scanning session. A typical contrast used in our analysis for instance would be "activity during the probe phase for six items collapsed across probe types and experimental blocks versus activity in the ITI blank-period". This method of time-series modeling and contrast estimation at each voxel reduces the number of images to one per subject per condition. To account for gain differences between fMRI sessions, activation values were normalized by their voxel averages. The resulting parametric maps images were smoothed using an isotropic Gaussian kernel (FWHM $=8$ $\mathrm{mm}$ ) and used as the data in the subsequent analysis. They contained 115 resolution elements as indicated by SPM99. These parametric maps serve as the dependent variables for the subsequent population-level multivariate analysis.

\subsection{Multivariate analysis}

Ordinal Trend Canonical Variates Analysis (OrT CVA) [22-24] was performed on the data. This analysis is similar to other regional covariance analyses techniques, notably Partial Least Squares, to the extent that it applies principal components analysis (PCA) to the data matrix that is transformed using a matrix representing the experimental design $[1,32,46]$. OrT CVA was designed to identify a covariance pattern in the MR signal utilizing each voxel, the expression of which decreases for as many subjects as possible from PRE to POST sleep deprivation.

It is important to stress the difference between our and other multivariate techniques: The latter might arrive at several covariance patterns - one per condition - by analyzing the across-subjects variances separately for each condition. These analyses usually establish differences between task conditions concerning: (1) the brain areas involved, i.e., brain area $X$ 's activation might account for a lot of the variance in task condition 1 , but not play a significant role in task condition 2, and vice versa; (2) a changing strength of the correlation between brain areas that are involved in both tasks, i.e., brain areas $X$ and $Y$ show a correlation in their activation across subjects of $R=0.8$ during task condition 1 , but this correlation reduces and switches directionality to $R=-0.1$ during task condition 2 .

Rather than examining differences in functional connectivity between conditions, we were interested in changes in 
regional activation induced by sleep deprivation that keep the functional connectivity unchanged from PRE to POST, and could therefore be captured in one covariance pattern. In our case, a single covariance pattern represents set of brain regions the connections of which are not changing from PRE to POST. Rather, subject expression of the covariance pattern varies from subject to subject with the additional constraint of a decrease from PRE to POST for as many subjects as possible. This means that most people are showing the same mutually correlated regional activation and de-activation in response to sleep deprivation, with individual differences in the degree of these changes.

The property of a systematic within-subjects change of pattern expression across task conditions (beyond mere mean trends) is called an "ordinal trend". The number of subjects who violate the rule of decreasing expression from PRE to POST can be used as a statistic to test the null hypothesis of the absence of an ordinal trend in the data [22]. Monte Carlo simulations of regional noise that is independently and identically distributed according to a Gaussian generate the $p$ level for the value of the numberof-exception criterion observed in our subject sample. For these simulations we used 18 subjects and 115 regional resolution elements in accordance with our experimental parameters. A significant ordinal trend lends additional credence to the claim that an activation pattern was obtained through the experimental design manipulation (= sleep deprivation in this case), rather than a significant change on the mean across conditions that might have come about as a result of overly influential subject outliers.

Activation patterns resulting from multivariate analysis assign different weights to all voxels included in the analysis, depending on the salience of their covariance contribution. Voxel weights that are positive indicate a positive correlation between the subject expression value and the associated regional activation, whereas negative weights indicate a negative correlation. This means that as the expression of a pattern increases, activation in the positively weighted regions increases as well, whereas activation in the negatively weighted regions decreases. The absolute magnitude of a regional weight determines the slope of this change: for instance, a region whose weight is twice is large as that of another also changes its activation twice as steeply. Whether a voxel weight is reliably different from zero is assessed by a bootstrap estimation procedure [17]. Denoting the point estimate of a voxel weight as $w$ and the standard deviation resulting from the bootstrap resampling procedure as $s_{w}$, we can assigned an inverse coefficient of variation (ICV) according to ICV $=w / s_{w}$. Sufficiently small variability of a voxel weight around its point estimate value in the resampling processes results in an ICV value of large magnitude and indicates a reliable contribution to the covariance pattern. As the threshold criterion, we chose $|\mathrm{ICV}|>3.5$; under the assumptions of a standard-normal distribution, this corresponds to a one-tailed probability of 0.0002 . If we take the number of resolution elements that characterizes the degree of spatial correlation caused by the smoothing process in our data analysis $(=115)$ the true $p$ value would be $0.0002 \times 115=0.0268$. The effects induced by sleep deprivation described in the Results are therefore strong enough to survive a multiple-comparison correction.

Individual subject's expression of the activation pattern during the PRE and POST sessions is quantified with the subject-scaling factor (SSF). The SSF is obtained by the operation of an inner product (= covariance across brain regions) between the covariance pattern in question and a subject's task scan. ${ }^{1}$ It quantifies to what extent a subject expresses the activation pattern in a task scan with a single number, which can be used for further analysis. Change in pattern expression for each subject as a function of sleep deprivation was measured by the PRE-POST difference of that subject's SSFs.

Once an activation pattern was identified that systematically decreased in expression as a function of sleep deprivation, we examined the correlation between individual change in network expression from PRE to POST sleep deprivation and change in their scores on the task performance measures.

\section{Results}

\subsection{Behavioral performance}

In addition to recognition accuracy and (within-subjects) mean reaction times, which are both commonly used to characterize the DMS-task performance, we also included (within-subjects) reaction time variability (standard deviation) and the rate of non-responses (for which subjects failed to respond in the allotted 3-s window during the probe period) in our behavioral outcome measures. This was prompted by the observation that subjects showed increased intra-individual variability in their reaction times as well as markedly increased non-response rates a function of sleep deprivation.

We subjected all four behavioral variables to a two-way ANOVA with sleep deprivation status, set size of stimulus and an interaction term as within-subjects factors. For the mean reaction times we found an effect of sleep deprivation $(F(1,17)=42.23, p<0.0001)$, an effect of set size $(F(2$, $34)=44.49, p<0.0001$ ), but no interaction between the two $(F(2,34)=0.78, p=0.47)$. For the STD of the reaction times, we found an effect of sleep deprivation $(F(1,17)=60.88$, $p<0.0001)$, no effect of set size $(F(2,34)=1.66, p=0.20)$, but a significant interaction $(F(2,34)=3.48, p<0.05)$. Look-

\footnotetext{
${ }^{1}$ For people who like to think in geometrical terms, the following analogies might be easier to understand: the activation pattern is a vector $\left(\boldsymbol{v}_{1}\right)$ in an $N$-dimensional vector space ( $N=$ number of brain regions) with Euclidean norm $=1$. An individual task scan is another vector in this $N$ dimensional space $\left(\boldsymbol{v}_{2}\right)$. The subject expression is the dot product between the two vectors, $\boldsymbol{v}_{1} \cdot \boldsymbol{v}_{2}$, and can be interpreted as the projection of $\boldsymbol{v}_{2}$ along $\boldsymbol{v}_{1}$
} 

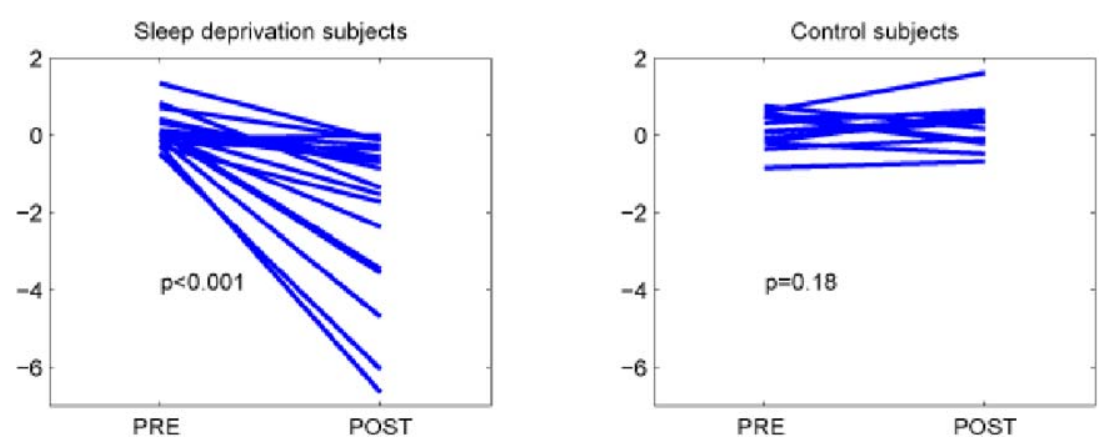

Fig. 2. Subject expression of the first principal component pattern resulting from the Ordinal Trend Canonical Variates Analysis. (Left) Sleep deprivation subjects: 17 of 18 subjects decreased their expression of the activation pattern $(p<0.001)$ as the result of sleep deprivation. (Right) Forward application of the sleep-deprivation pattern to control subjects: No systematic decrease was observed, and four people increased their expression of the covariance pattern $(p=0.18)$.

ing at the post hoc contrasts more carefully reveals that this interaction is due to a bigger sleep-deprivation-induced difference of the STD values for three stimulus items than for one or six stimulus items. For the recognition accuracy, we found an effect of sleep deprivation $(F(1,17)=16.6$, $p<0.005)$, but no effect of set size $(F(2,34)=1.31$, $p=0.29)$, and no interaction $(F(2,34)=0.11, p=0.89)$. We also looked at the overall non-response rate as a function of sleep deprivation and set size and obtained an effect of sleep deprivation $(F(1,17)=59.69, p<0.0001)$, but no effect of set size $(F(2,34)=1.43, p=0.25)$, and no interaction $(F(2$, $34)=1.65, p=0.21)$.

Since the set-size 6 condition is the one used to generate the activation patterns in the OrT CVA, the behavioral variables that pertain to a stimulus array size of six items are of special interest. On the first day of the study, prior to any sleep deprivation, recognition accuracy was near ceiling $(96.5 \pm 3.8 \%)$. Within-subjects mean reaction time was $1190 \pm 198 \mathrm{~ms}$. Intra-individual variability (STD within subject) was $347 \pm 115 \mathrm{~ms}$. The fraction of nonresponses (when subjects exceeded the 3 -s deadline) was very low at $0.03+0.10 \%$. There was a negative effect of sleep deprivation on performance. On day 2 of the study, after $48 \mathrm{~h}$ of sleep deprivation, recognition accuracy was reduced and more variable across subjects $(82.6 \pm 17.0 \%)$, mean reaction time was increased $(1486 \pm 296 \mathrm{~ms})$, intraindividual reaction time variability was increased (557 \pm $141 \mathrm{~ms}$ ), and the fraction of non-responses was increased $(30.11 \pm 18.74 \%)$. All measures show highly significant differences as a function of sleep deprivation in pairedsample $t$ tests (17 degrees of freedom), yielding one-tailed $p$ values $<0.001$.

\section{2. fMRI data}

For the purposes of the MR image analysis, our experiment contained two design parameters: sleep deprivation status (PRE/POST) and memory load (one, three or six items). Motivated by the observation that the behavioral variables in our experiment did not show any reliable interaction-effect between these two factors, we hypothesized that the effects of those two factors on neural activation patterns during retrieval would also be non-interacting. Furthermore, regardless of an interaction, we reasoned that the effects of sleep deprivation on the neural activation patterns would be stronger than the effect of memory load, causing us to focus primarily on the effects of sleep deprivation. In order to allow for a possible interaction, however, we did not collapse our analysis across different memory loads, but decided to identify sleep-deprivation-induced changes at the most demanding load level. ${ }^{2}$

We therefore subjected only the data from the probe phase for six stimulus items to an Ordinal Trend Analysis. ${ }^{3}$ The fMRI data from non-response trials were excluded from any further analysis. The first principal component of the OrT CVA (accounting for 30\% of the variance) displayed ordinal trend properties. Seventeen of 18 subjects decreased their pattern expression ( $p<0.001$; see Fig. 2), suggesting an unambiguous neural correlate of sleep deprivation. Because the well-rested scans always preceded the scans immediately following sleep deprivation, we verified further that the activation differences associated with this pattern were due to sleep deprivation only, and not due to an order effect. We applied the sleep-deprivation-related pattern to the data of 14 control subjects that were scanned while performing the DMS task $48 \mathrm{~h}$ apart without undergoing sleep deprivation, and did not find any systematic increase

\footnotetext{
2 We also applied Ordinal Trend Analysis to the data from all probe phases (1, 3 and 6$)$ on day 1 in order to identify an activation pattern whose subject expression increases as a function of memory load. We failed to find such a pattern (best-fit: expression of PC $1-4$ yields an activation pattern with five exceptions, $p=0.39$ ), indicating that a load modulation in activation does not contribute enough variance to be detected in a covariance decomposition of the data from our subject sample.

3 We also subjected the data from stimulus and retention phase for six memorized items to an Ordinal Trend analysis, similarly to the treatment of the data from the probe phase, but we did not yield a significant effect of sleep deprivation that correlated with any of the behavioral measures.
} 
or decrease in pattern expression from scan 1 to scan 2 for the control subjects, thus ruling out that order effects gave rise to the activation pattern in the first place. Furthermore, the subject expression of the sleep-deprivation pattern does not differ significantly between the groups in the well-rested state, (subjects on day 1 vs. controls on day 1: $p=0.95$, subjects on day 1 vs. controls on day $2: p=0.73 ; p$ levels from two-tailed $t$ tests.).

Brain regions that concomitantly decreased in activation (as ascertained by the bootstrap test) for the majority of subjects as a function of sleep deprivation were found mainly in posterior areas, particularly visual association (ventral stream) areas and parietal areas (BA 7 and BA 40). Brain regions that concomitantly increased in activation for the majority of subjects were found in the anterior cingulate gyrus (BA 32), thalamus and basal ganglia as well the anterior lobe of the cerebellum (Figs. 3 and 4; Tables 1 and 2).

We then tested whether individuals' pattern expression correlated with individual's behavioral performance and found that the decrease in expression as a result of sleep deprivation was significant in predicting the drop in recognition accuracy $\left(R^{2}=0.27, p<0.05\right)$ from day 1 to day 2 (Fig. 5).
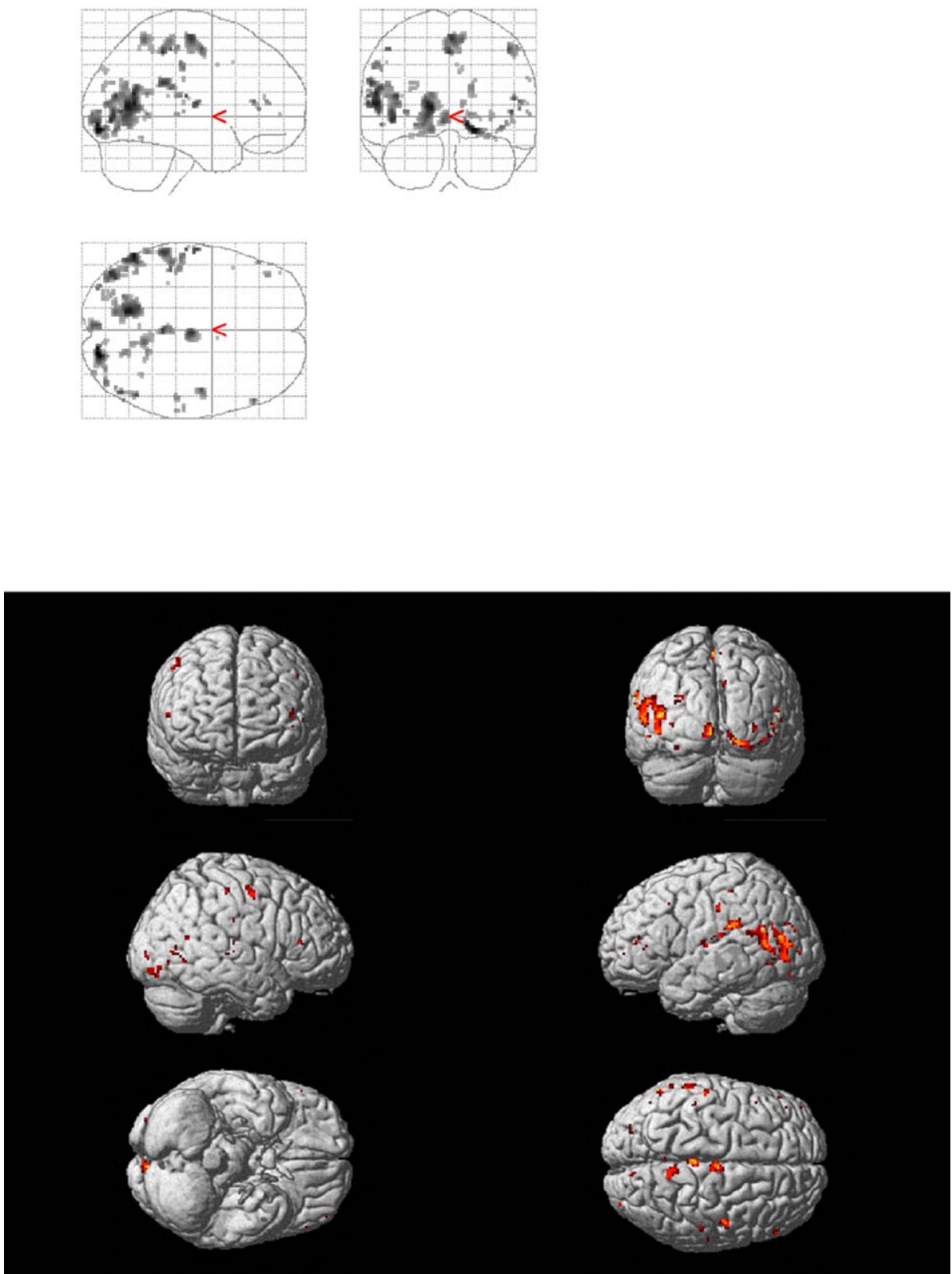

Fig. 3. (Upper half) Axial, coronal and sagittal glass-brain projections of brain regions that have positive weights in the activation pattern, i.e., whose associated activation decreases for most subjects from PRE to POST. The threshold of the ICV statistic was $>3.5$. (Lower half) Surface-rendered projections of the regions displayed in the glass-brain projections. 
Furthermore, the decrease in the activation pattern's expression predicted the increase in intra-individual reaction variability from PRE to POST $\left(R^{2}=0.59, p<0.0005\right)$ (Fig. 6).

The drop in recognition accuracy and the increase in intra-individual reaction time variability were correlated $\left(R^{2}=0.31, p<0.05\right)$. When the influence of the recognition accuracy drop was removed from the increase in intra-individual reaction time variability, the correlation with the change in the covariance pattern's expression from PRE to POST was reduced, but still significant with the same sign of the correlation as before $\left(R^{2}=0.33\right.$, $p<0.05)$.
The decrease in pattern expression from PRE to POST also correlated significantly with the increase in the number of non-responses from PRE to POST $\left(R^{2}=0.45, p<0.005\right.$, figure not shown). That is, the expression of the covariance pattern during the epochs in which subjects made responses could predict the global rate of non-responses. This correlation though became non-significant when the influence of the increase in reaction time variability was partialled out from the increase in lapses.

An additional observation about the correlation between changes in behavioral performance variables and pattern expression is that this correlation is mainly driven by the POST condition. Pattern expression during POST correlates
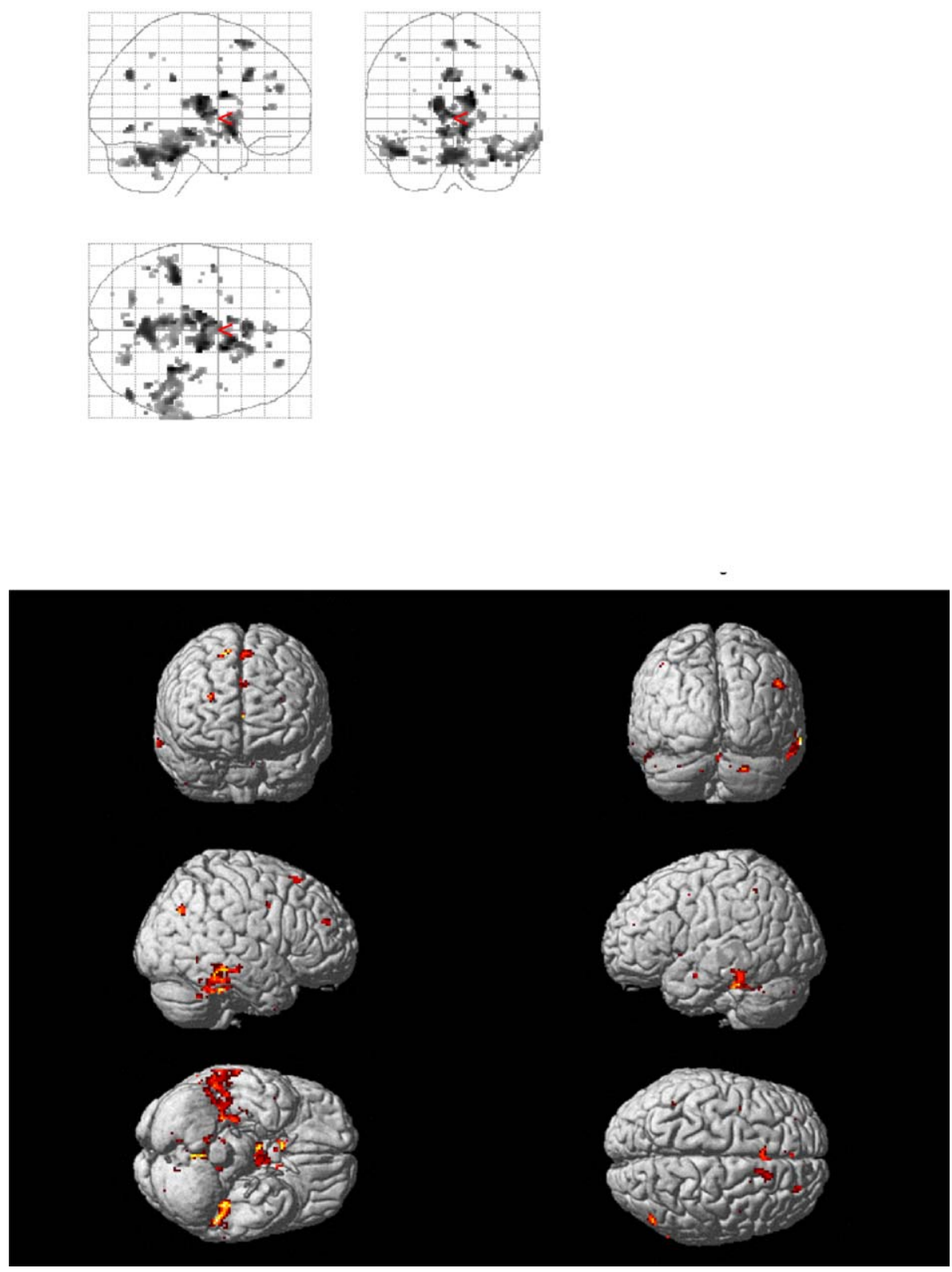

Fig. 4. (Upper half) Axial, coronal and sagittal glass-brain projections of brain regions that have negative weights in the activation pattern, i.e., whose associated activation increases for most subjects from PRE to POST. The threshold of ICV statistic was $<-3.5$. (Lower half) Surface-rendered projections of the regions displayed in the glass-brain projections. 
Table 1

Talairach locations of brain regions with significant de-activation from PRE to POST as ascertained by a bootstrap resampling test (ICV $>3.5)$ (Talairach Daemon Client 1.1, Research Imaging Center, University of Texas Health Science Center at San Antonio)

\begin{tabular}{|c|c|c|c|c|c|}
\hline$X$ & $Y$ & $Z$ & & & \\
\hline-14 & -62 & 10 & Posterior Cingulate & Gray Matter & $\begin{array}{l}\text { Brodmann } \\
\text { area } 30\end{array}$ \\
\hline-55 & -58 & 12 & $\begin{array}{l}\text { Middle Temporal } \\
\text { Gyrus }\end{array}$ & Gray Matter & $\begin{array}{l}\text { Brodmann } \\
\text { area } 39\end{array}$ \\
\hline-40 & -77 & 13 & $\begin{array}{l}\text { Middle Occipital } \\
\text { Gyrus }\end{array}$ & Gray Matter & $\begin{array}{l}\text { Brodmann } \\
\text { area } 19\end{array}$ \\
\hline 2 & -13 & 56 & $\begin{array}{l}\text { Medial Frontal } \\
\text { Gyrus }\end{array}$ & $*$ & $*$ \\
\hline-59 & -34 & 26 & $\begin{array}{l}\text { Inferior Parietal } \\
\text { Lobule }\end{array}$ & Gray Matter & $\begin{array}{l}\text { Brodmann } \\
\text { area } 40\end{array}$ \\
\hline-51 & -30 & 22 & $\begin{array}{l}\text { Inferior Parietal } \\
\text { Lobule }\end{array}$ & Gray Matter & $\begin{array}{l}\text { Brodmann } \\
\text { area } 40\end{array}$ \\
\hline 48 & -68 & 7 & $\begin{array}{l}\text { Middle Occipital } \\
\text { Gyrus }\end{array}$ & $*$ & $*$ \\
\hline 50 & -56 & 16 & $\begin{array}{l}\text { Superior Temporal } \\
\text { Gyrus }\end{array}$ & Gray Matter & $\begin{array}{l}\text { Brodmann } \\
\text { area } 22\end{array}$ \\
\hline 46 & -5 & 48 & Precentral Gyrus & Gray Matter & $\begin{array}{l}\text { Brodmann } \\
\text { area } 6\end{array}$ \\
\hline 10 & -48 & 58 & Precuneus & Gray Matter & $\begin{array}{l}\text { Brodmann } \\
\text { area } 7\end{array}$ \\
\hline 4 & -42 & 48 & Precuneus & * & $*$ \\
\hline 40 & -80 & -3 & $\begin{array}{l}\text { Inferior Occipital } \\
\text { Gyrus }\end{array}$ & Gray Matter & $\begin{array}{l}\text { Brodmann } \\
\text { area } 19\end{array}$ \\
\hline-34 & -83 & 8 & $\begin{array}{l}\text { Middle Occipital } \\
\text { Gyrus }\end{array}$ & Gray Matter & $\begin{array}{l}\text { Brodmann } \\
\text { area } 19\end{array}$ \\
\hline-51 & -62 & -2 & $\begin{array}{l}\text { Inferior Temporal } \\
\text { Gyrus }\end{array}$ & Gray Matter & $\begin{array}{l}\text { Brodmann } \\
\text { area } 19\end{array}$ \\
\hline 53 & -66 & 9 & $\begin{array}{l}\text { Middle Temporal } \\
\text { Gyrus }\end{array}$ & Gray Matter & $\begin{array}{l}\text { Brodmann } \\
\text { area } 37\end{array}$ \\
\hline 16 & -48 & 4 & $\begin{array}{l}\text { Parahippocampal } \\
\text { Gyrus }\end{array}$ & Gray Matter & $\begin{array}{l}\text { Brodmann } \\
\text { area } 30\end{array}$ \\
\hline-42 & 41 & 9 & $\begin{array}{l}\text { Inferior Frontal } \\
\text { Gyrus }\end{array}$ & Gray Matter & $\begin{array}{l}\text { Brodmann } \\
\text { area } 46\end{array}$ \\
\hline 32 & -89 & 4 & $\begin{array}{l}\text { Middle Occipital } \\
\text { Gyrus }\end{array}$ & Gray Matter & $\begin{array}{l}\text { Brodmann } \\
\text { area } 18\end{array}$ \\
\hline 8 & -78 & 35 & Cuneus & Gray Matter & $\begin{array}{l}\text { Brodmann } \\
\text { area } 19\end{array}$ \\
\hline 59 & -20 & 29 & Postcentral Gyrus & Gray Matter & $\begin{array}{l}\text { Brodmann } \\
\text { area } 2\end{array}$ \\
\hline-4 & -52 & 50 & Precuneus & * & $*$ \\
\hline 57 & -21 & 12 & $\begin{array}{l}\text { Transverse Temporal } \\
\text { Gyrus }\end{array}$ & Gray Matter & $\begin{array}{l}\text { Brodmann } \\
\text { area } 41\end{array}$ \\
\hline-44 & 50 & -1 & $\begin{array}{l}\text { Middle Frontal } \\
\text { Gyrus }\end{array}$ & Gray Matter & $\begin{array}{l}\text { Brodmann } \\
\text { area } 10\end{array}$ \\
\hline 6 & 6 & 37 & Cingulate Gyrus & Gray Matter & $\begin{array}{l}\text { Brodmann } \\
\text { area } 24\end{array}$ \\
\hline-48 & 17 & 38 & $\begin{array}{l}\text { Middle Frontal } \\
\text { Gyrus }\end{array}$ & Gray Matter & $\begin{array}{l}\text { Brodmann } \\
\text { area } 8\end{array}$ \\
\hline
\end{tabular}

White matter locations have been removed.

with behavioral performance during POST for two of the three behavioral variables (recognition accuracy: $R^{2}=0.18$, $p=0.08$; STD RT6: $R^{2}=0.50, p<0.001$; non-response rate: $\left.R^{2}=0.44, p<0.005\right)$. Higher subject expression of the sleepdeprivation pattern during POST implies better performance, consistent with the earlier observation that the more subjects decrease their pattern expression from PRE to
Table 2

Talairach locations of areas with significant activation from PRE to POST as ascertained by a bootstrap resampling test $(\mathrm{ICV}<-3.5)$

\begin{tabular}{|c|c|c|c|c|c|}
\hline$X$ & $Y$ & $Z$ & & & \\
\hline 14 & 7 & 18 & Caudate & Gray Matter & $\begin{array}{l}\text { Caudate } \\
\text { Body }\end{array}$ \\
\hline 16 & -13 & 12 & Thalamus & Gray Matter & $\begin{array}{l}\text { Ventral } \\
\text { Lateral } \\
\text { Nucleus }\end{array}$ \\
\hline-14 & -13 & 8 & Thalamus & Gray Matter & $\begin{array}{l}\text { Ventral } \\
\text { Lateral } \\
\text { Nucleus }\end{array}$ \\
\hline 10 & -9 & 15 & Thalamus & Gray Matter & $\begin{array}{l}\text { Anterior } \\
\text { Nucleus }\end{array}$ \\
\hline 30 & -36 & -25 & Culmen & $*$ & $*$ \\
\hline-40 & -32 & -20 & $\begin{array}{l}\text { Parahippocampal } \\
\text { Gyrus }\end{array}$ & Gray Matter & $\begin{array}{l}\text { Brodmann } \\
\text { area } 36\end{array}$ \\
\hline 4 & 7 & -15 & $\begin{array}{l}\text { Medial Frontal } \\
\text { Gyrus }\end{array}$ & $*$ & $*$ \\
\hline-4 & 25 & 30 & Cingulate Gyrus & Gray Matter & $\begin{array}{l}\text { Brodmann } \\
\text { area } 32\end{array}$ \\
\hline 4 & 21 & 27 & Cingulate Gyrus & $*$ & $*$ \\
\hline-2 & 22 & 52 & $\begin{array}{l}\text { Superior Frontal } \\
\text { Gyrus }\end{array}$ & Gray Matter & $\begin{array}{l}\text { Brodmann } \\
\text { area } 8\end{array}$ \\
\hline-8 & 8 & 1 & Caudate & Gray Matter & $\begin{array}{l}\text { Caudate } \\
\text { Head }\end{array}$ \\
\hline-4 & -66 & -3 & Culmen & $*$ & $*$ \\
\hline 42 & -54 & -28 & Tuber & * & $*$ \\
\hline 24 & 48 & 20 & $\begin{array}{l}\text { Superior Frontal } \\
\text { Gyrus }\end{array}$ & Gray Matter & $\begin{array}{l}\text { Brodmann } \\
\text { area } 10\end{array}$ \\
\hline 12 & 40 & 18 & $\begin{array}{l}\text { Medial Frontal } \\
\text { Gyrus }\end{array}$ & Gray Matter & $\begin{array}{l}\text { Brodmann } \\
\text { area } 9\end{array}$ \\
\hline-48 & -54 & -23 & Tuber & $*$ & $*$ \\
\hline-24 & -65 & -25 & Uvula & * & $*$ \\
\hline 24 & -69 & -27 & Pyramis & $*$ & $*$ \\
\hline 14 & -69 & -25 & Uvula & $*$ & $*$ \\
\hline 22 & -77 & -25 & Uvula & $*$ & $*$ \\
\hline 32 & -14 & -16 & $\begin{array}{l}\text { Parahippocampal } \\
\text { Gyrus }\end{array}$ & Gray Matter & Hippocampus \\
\hline 63 & -55 & -2 & $\begin{array}{l}\text { Middle Temporal } \\
\text { Gyrus }\end{array}$ & Gray Matter & $\begin{array}{l}\text { Brodmann } \\
\text { area } 37\end{array}$ \\
\hline 24 & 12 & 1 & Lentiform Nucleus & Gray Matter & Putamen \\
\hline 2 & -52 & -1 & Culmen & $*$ & $*$ \\
\hline 12 & -50 & -34 & Cerebellar Tonsil & $*$ & $*$ \\
\hline-22 & 10 & -4 & Lentiform Nucleus & Gray Matter & Putamen \\
\hline 24 & -32 & -12 & $\begin{array}{l}\text { Parahippocampal } \\
\text { Gyrus }\end{array}$ & Gray Matter & $\begin{array}{l}\text { Brodmann } \\
\text { area } 36\end{array}$ \\
\hline 57 & -53 & -18 & Fusiform Gyrus & Gray Matter & $\begin{array}{l}\text { Brodmann } \\
\text { area } 37\end{array}$ \\
\hline-46 & -48 & -23 & Culmen & $*$ & $*$ \\
\hline-10 & -73 & -27 & Pyramis & $*$ & $*$ \\
\hline-42 & -48 & 48 & $\begin{array}{l}\text { Inferior Parietal } \\
\text { Lobule }\end{array}$ & Gray Matter & $\begin{array}{l}\text { Brodmann } \\
\text { area } 40\end{array}$ \\
\hline 10 & -64 & -27 & Uvula & $*$ & $*$ \\
\hline-65 & -34 & -10 & $\begin{array}{l}\text { Middle Temporal } \\
\text { Gyrus }\end{array}$ & Gray Matter & $\begin{array}{l}\text { Brodmann } \\
\text { area } 21\end{array}$ \\
\hline-24 & -44 & -18 & Culmen & $*$ & $*$ \\
\hline-48 & -58 & -24 & Tuber & $*$ & $*$ \\
\hline 20 & 12 & 51 & $\begin{array}{l}\text { Superior Frontal } \\
\text { Gyrus }\end{array}$ & Gray Matter & $\begin{array}{l}\text { Brodmann } \\
\text { area } 6\end{array}$ \\
\hline-28 & -81 & -23 & Uvula & * & $*$ \\
\hline
\end{tabular}

White matter locations have been removed. 


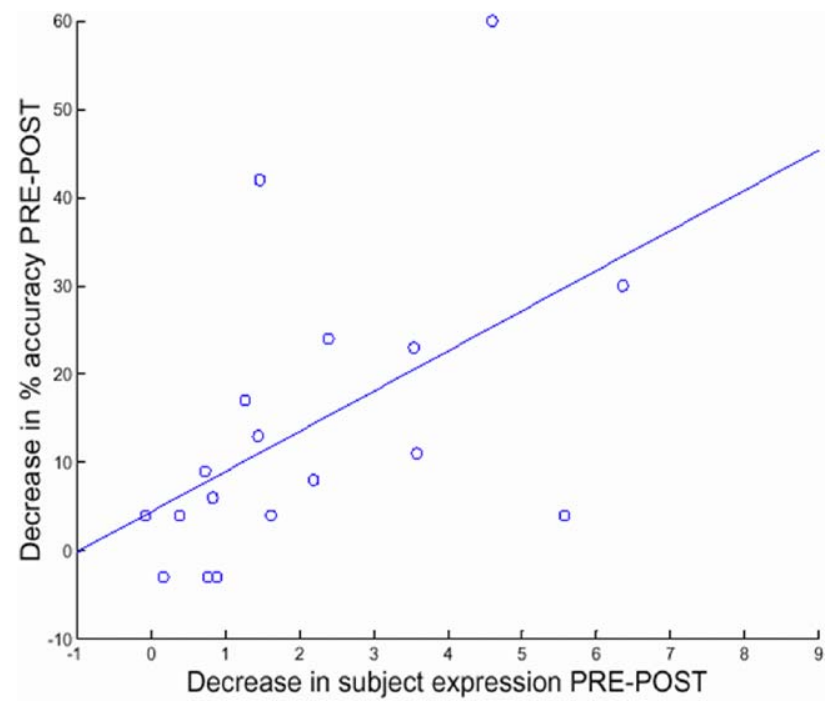

Fig. 5. Brain-behavior correlation of pattern expression and worsening recognition accuracy due to sleep deprivation. The bigger the subjects' decrease of their pattern expression, the worse their drop in recognition accuracy $\left(R^{2}=0.27, p<0.05\right)$.

POST, the worse their performance decrement. During PRE, subject expression of the sleep-deprivation pattern did not bear any relationship to performance.

Although the decrease in expression of the activation pattern did not correlate with the decrease in (within-subjects) mean reaction times as a result of sleep deprivation, these brain-behavior correlations provide additional confirmation of having established a true neural correlate of sleep deprivation and its effects on cognitive performance. Individual decreases in subject expression of the activation pattern could thus predict the degree to which recognition accuracy worsened as well as the degree to which intraindividual reaction time variability increased. It could not predict the degree to which subjects slowed down as a result of sleep deprivation. Further, the degree of slowing could not be predicted well by a combined expression of the more inclusive set of the first six principal components in a linear regression $\left(R^{2}=0.15\right)$, demonstrating that if a neural correlate of slowing exists and contributes enough variance to be captured by a PCA, it will probably be found during the retention or stimulus phase (to be described in a future report).

Activation patterns resulting from a multivariate analysis of one data set can be applied prospectively to different data sets in order to prove or disprove the patterns' utility in accounting for experimental variables with its subject expression. We decided to exploit this feature and apply the sleep deprivation pattern to the data from the probe phases for one and three stimulus items. Pattern expression still showed a decrease from PRE to POST for the majority of subjects with three exceptions for both stimulus array sizes of 1 and $3(p<0.01)$. For the array size of 1 , decrease in pattern expression still correlated with the increase in intra- individual reaction time variability $\left(R^{2}=0.34, p<0.05\right)$; for array size 3 , decrease in pattern expression still correlated with a decrease in recognition accuracy $\left(R^{2}=0.24, p<0.05\right)$ (graphs not shown) (Fig. 7).

The preserved relationship between change in pattern expression and sleep deprivation for all stimulus array sizes suggests the absence of any interaction between the effects of memory load and sleep deprivation. For further substantiation, we forward-applied the previously derived sleep deprivation pattern to the probe phase data. The resulting values represent the degree to which the sleep-deprivation pattern was expressed in each subject in each set-size condition both on day 1 and day 2 . These values were into a two-way ANOVA that contained sleep deprivation status, memory load and an interaction term as factors, similarly to our analyses in the Behavioral Performance. Similarly to the behavioral variables, we found an effect of sleep deprivation $(F(1,17)=21.24, p<0.0001)$ and an effect of memory load $(F(2,34)=4.66, p<0.05)$, but no interaction $(F(2,34)=$ $3.13, p=0.07)$.

The regions involved in the obtained activation pattern point to involvement of a spatial attentional network (frontal and parietal regions) as well as early perceptual processing (occipital regions; cf. Ref. [28]). We also applied the activation pattern to the data from the stimulus phase for six items of the DMS task. The PRE-POST difference in subject expression of the pattern in this phase of the task also displays a significant correlation with behavior that was observed during the probe phase. The decrease in expression correlates significantly with the increase in reaction time variability $\left(R^{2}=0.40, p<0.005\right)$, the increase in the non-response rate $\left(R^{2}=0.33, p<0.05\right)$, and marginally with

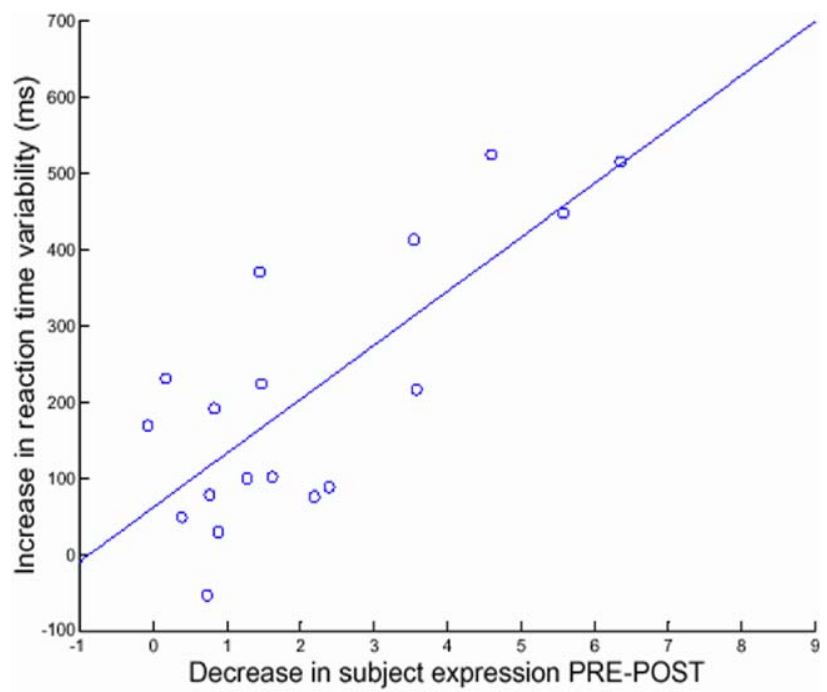

Fig. 6. Brain-behavior correlation of pattern expression and increased intra-individual reaction time variability due to sleep deprivation. The bigger the subjects' decrease of their pattern expression, the larger their increase in reaction time variability as measured by the within-subjects standard deviation $\left(R^{2}=0.59, p<0.0005\right)$. 

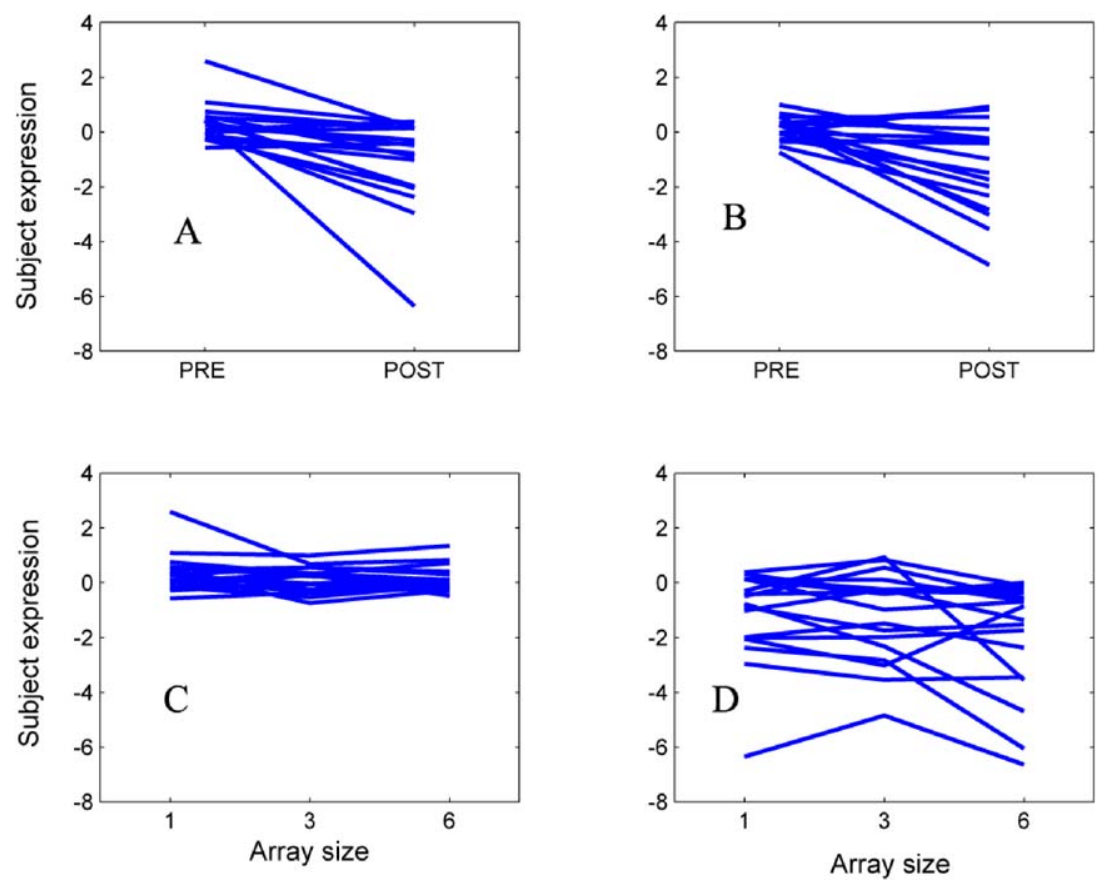

Fig. 7. Prospective application of the sleep deprivation pattern obtained from the probe phase with six items to subject data from probe phases for one and three items. (A) Subject expression of the sleep deprivation pattern for the probe phase with one stimulus item; 15 of 18 subjects decrease their subject expression of the forwardly applied pattern $(p<0.01)$ from PRE to POST; (B) subject expression of the sleep deprivation pattern for the probe phase with three stimulus items; 15 of 18 subjects decrease their expression of the forwardly applied pattern $(p<0.01)$ from PRE to POST. (C) Plot of the of subject expression of the sleep deprivation pattern for all probe phases on day 1 of the study, prior to sleep deprivation, as a function stimulus array size: To illustrate the within-subjects behavior, expression values at the three different stimulus size levels were connected by a line for each subject. (D) Plot of the of subject expression of the sleep deprivation pattern for all probe phases on day 2 of the study, after to sleep deprivation, as a function stimulus array size. In a two-way ANOVA, there was an effect of sleep deprivation and memory load, but no interaction.

the decrease in recognition accuracy $\left(R^{2}=0.18, p=0.08\right)$. The PRE-POST differences in pattern expression in the two task phases are also strongly related to each other $\left(R^{2}=0.56\right.$, $p<0.0005)$.

Forward application to the data from the retention phase is also possible, albeit not plausible on account of the different cognitive mechanisms operational during that phase. For completeness, we decided to apply the pattern to the data from this phase also, but predictably did not achieve any correlation between subject expression and any of the behavioral measures.

\section{Discussion}

We successfully identified a sleep-deprivation-related activation pattern in the probe data from a delayedmatch-to-sample task. Subject expression of this activation pattern decreased as a function of sleep deprivation for 17 out of 18 subjects $(p<0.001)$, and correlated significantly with performance measures that were degraded by sleep deprivation. That is, the more subjects decreased their expression of this pattern, the worse their recognition accuracy became, the more their number missed responses increased, and the more their intra- individual variability in reaction time increased as a function of sleep deprivation.

Brain regions in this pattern whose associated activation decreased through the course of sleep deprivation were mainly found in the areas of the "ventral" and "dorsal stream" of early visual processing system, i.e., occipital and temporal regions (BA 18, 19, 37, 38, 39) as well as parietal regions (BA 40). Some of these brain regions were identified in previous cognitive neuroimaging experiments involving visual memory and novelty processing tasks of objects' features and imagery $[9,11,18,34,36,40]$. Activation in these areas and the precuneus (BA 7) and the inferior parietal lobule (BA 40), areas associated with dorsal stream activity previously identified in processing object location $[5,7,8]$, decreased as a function of sleep deprivation for the majority of subjects in our experiment. These results suggest that sleep deprivation may affect the retrieval of previously encoded objects by disrupting perceptual processing when comparing the probe to the remembered items. Most subjects cannot sustain activation in these brain regions after sleep deprivation, and consequently their performance suffers. (Involvement of pre-frontal and frontal brain regions was limited, although present in BA $6,8,10$ and 46 , and is discussed below.) 
Regions whose associated activation increased as a function of sleep deprivation were found mainly in the basal ganglia (caudate head, putamen) and thalamus as well as the anterior cingulate gyrus (BA 32) and the right superior and medial frontal gyri (BA 9, 10). Activation of these regions in concert, resulting in high covariance, is plausible on the basis of their anatomical connectivity that has been investigated in macaque monkeys through anatomical tract-tracer studies [31]. The basal ganglia project to a variety or motor and pre-motor brain regions via thalamic relay nuclei. The ventral-anterior nucleus projects to anterior cingulate gyrus, consistent with our finding. Several neuroimaging and computational modeling studies have elucidated this region's involvement in evaluative processes like monitoring response conflict, e.g., Refs. $[4,6,29]$, affording the possible interpretation that sleep deprivation causes increased monitoring demand in the selection of the appropriate response to the probe letter on display.

As concerns the PFC, we noted activation with small spatial extent that passed the bootstrap threshold criterion. Bilateral regions in dorsal PFC (inferior frontal gyri; BA 46) showed a sleep-deprivation-related de-activation that was predictive of worsening recognition performance. This finding is consistent with recent event-related studies of the DMS task $[39,42]$, which ascribed modulation by memory load and task performance (recognition accuracy) to the activation in dorsal PFC during the probe phase. We can therefore refine the statement of the independence between the experimental factors sleep deprivation and memory load: They might give rise to different activation patterns whose subject expression values are dissociated from each other, yet, individual brain regions can be influenced by both simultaneously in a non-interactive manner. Thus, activation in dorsal PFC is increased by increasing memory load, but decreased by sleep deprivation.

To our knowledge, multivariate techniques have so far not been applied to sleep deprivation and only rarely been applied to working memory tasks [21,33]. These studies investigated the effects of the delay between encoding and retrieval as well as subjects' age differences on the ongoing activity during the delay period in a face-matching task. Path analyses quantified the strength of interaction between different key brain areas as a function of the length of the delay period. However, because of their different design goals compared with our study, it is difficult to see exactly how their findings would inform the analysis in this paper.

The regions found to de-activate in our event-related fMRI study are broadly in line with regions that showed decreased glucose metabolism in an FDG-PET study [45] and an earlier fMRI study [14] that both contrasted neural performance on a serial addition/subtraction task before and after 24 and $35 \mathrm{~h}$ of sleep deprivation, respectively. Alertness and cognitive performance declined with de-activation in pre-frontal, parietal and thalamic regions.
Some of the regions showing increased activation as a result of sleep deprivation have also been identified in other studies: In a study of $24 \mathrm{~h}$ of sleep deprivation involving an attention task [38], the ventrolateral thalamus was also found to display increased activation, as was the anterior cingulate in a study of $35 \mathrm{~h}$ of sleep deprivation involving an fMRI dual-task study of verbal learning and serial subtraction task [16]. The latter activation was also interpreted as arising from the increased need for monitoring response conflicts in the sleep-deprived state, similarly to our suggestion above.

On the other hand, the above dual-task study [16] as well an fMRI study by the same authors dealing with $35 \mathrm{~h}$ of sleep deprivation and a verbal learning task [15] found increased activation in the major regions of the frontal and parietal lobes that were decreasing in activation in the current report. This discrepancy shows the need for further investigation. A possible explanation is that the increased activation in the areas reported by these two studies after 35 $\mathrm{h}$ of sustained wakefulness cannot persist for $48 \mathrm{~h}$, and reverses its sign relative to the baseline. It is worth considering, though, that in addition to the degree of sleep deprivation, our study differed from these studies on two more counts: (1) the task: a DMS-task with single letters vs. a verbal learning task using words, (2) the data analysis: multivariate vs. univariate analysis. Factor (1) could plausibly bring about a difference in the behavior of the prefrontal cortex. Factor (2) could also result in the differences observed: Our analysis identifies frontal and parietal regions in the context of a covariance pattern. In these areas, there could be increasing activation in response to sleep deprivation that is not accounted for by the covariance pattern (because of a lack of sufficient variance in the principal components analysis), while the modulation of the covariance pattern might dictate de-activation for these areas.

Regardless of the precise mechanisms, it seems likely that the different nature of the tasks used in our and the other recent fMRI studies of sleep deprivation [14-16,45] accounts for some of the different behavior observed in frontal and parietal regions. Studies [14-16] all used 35 $\mathrm{h}$ of sleep deprivation, but employed arithmetic, verbal learning and a dual-task situation involving both, to obtain changes in activation of both positive and negative signs. Studies $[14,45]$ on the other hand used similar arithmetic tasks, but different amounts of sleep deprivation, and arrived at similar changes in activation, regardless of potential circadian confounds $[2,3,35]$ hampering the cross-study comparison.

In addition to a broad characterization of the cognitive processes contributing to the obtained sleep-deprivation pattern, we can speculate about which specific processes underlie our results. Expression of the sleep deprivation pattern during the probe phase was independent of the size of the stimulus array, which is consistent with the hypothesis that sleep deprivation does not affect memory scanning. 
Regardless of the number of items subject had to keep in mind, sleep deprivation caused the same noticeable decrease in the expression of the activation pattern. Likewise, regardless of whether subjects were sleep deprived or not when they performed the task, the activation pattern failed to evidence any modulation by memory load in its subject expression.

In addition to memory scanning, the DMS task probe phase requires binary decision, response selection and motor execution processes. If the binary decision process were impacted by sleep deprivation, one would expect the interaction between sleep deprivation status and probe type (true positive/true negative) to affect MR signal pattern expression in a two-factorial design. We did not have enough data to facilitate such an analysis, and can therefore neither confirm nor rule out that the binary decision process is affected by sleep deprivation. Extending this logic, we cannot infer anything about sleepdeprivation effects on response selection because this experiment contained no explicit manipulation that could interact with sleep deprivation to affect pattern expression. However, as noted above, involvement of the basal ganglia structures may in fact suggest changes to response selection. We can infer that the obtained sleepdeprivation-related activation pattern does not reflect changes to the motor execution processes due to the lack of correlation between pattern expression and the mean RT.

Our findings can be situated in a broader context of the effects of sleep deprivation that have been established in the sleep-deprivation literature. A thorough review article [13] conceptualized sleep deprivation as consisting of five major effects that might all be present to varying degrees: (1) cognitive slowing, (2) optimum response shifts, (3) lapsing, (4) memory decrements and (5) vigilance decrements (timeon-task effects). We can relate our results to some of these aspects.

Although we observed general cognitive slowing behaviorally (independent of memory scanning) in our experiment, we failed to locate any neural correlates in the major principal components of our analysis. Apparently, these effects do not contribute enough variance and are superseded by the other effects in the list. Based on the overall slowing of reaction time evident in our behavioral data, we might expect that shifts occurred in optimal responses as well. However, our data set was too small to analyze optimum response shifts since such an analysis requires a further subdivision of the data.

Lapsing accounted for a large part of our effects since expression of our covariance pattern correlates with the rate of non-responses and the reaction time variability in line with the above-mentioned review [13], which identified lapsing as a main cause of increased reaction time variability. This is also the likely cause of the colinearity between the rate of non-responses and reaction time variability shown in the Results.
With respect to memory decrements, our behavioral as well as neuroimaging results did not indicate that memory scanning per se was affected by sleep deprivation. However, sleep deprivation caused a decrease in overall performance accuracy that was correlated with network expression, directly implicating memory processing in general as a source of sleep-deprivation-related performance decrements.

Vigilance decrements due to sleep deprivation usually become apparent over the course of extended behavioral testing. Our behavioral task consisted of three 10-min blocks. We cannot examine vigilance decrements within blocks because of our randomized trial design. However, we can examine vigilance decrements across the three blocks. To test this possibility, our behavioral variables were subjected to a three-way ANOVA in which the block number, set size and the sleep deprivation status are entered as factors. We did not find any main effect of block number or interactions between block number (time-on-session) and sleep deprivation status for any of the variables. This indicates that reduced expression of the activation pattern after sleep deprivation is not due to reduced performance or attention to the task across the testing session. However, the possibility remains that vigilance decrements within a block (time-on-block) can partially account for the observed effects.

Despite this absence of time-on-task vigilance effects, the regions involved in the obtained activation pattern point to involvement of a spatial attentional network (frontal and parietal regions) as well as early perceptual processing (occipital regions; cf. Ref. [28]). Both spatial attention and early visual processing are important components of the stimulus phase of the task trials as well as the probe phase from which our activation pattern was obtained. To test this possibility, we applied the probe-period activation pattern to the data from the stimulus phase for six items of the DMS task. The PRE-POST difference in subject expression of the pattern in this phase of the task displays nearly the same correlation with behavior that was observed during the probe phase. The decrease in expression correlates significantly with the increase in reaction time variability and the non-response rate. The PRE-POST differences in pattern expression in the two task phases are also strongly related to each other. This finding supports the notion that the some cognitive operations reflected in the probe-period activation pattern are also present in the stimulus phase, and that the neural activation pattern obtained during probe is sufficient, but not necessary, to account for some of the behavioral decrements.

In summary, we established a robust neural correlate of sleep deprivation during the probe phase of a DMS task. Expression of the identified pattern was independent of memory load, and accounted for worsening task performance as reflected by decreased recognition accuracy, increased non-response rate and increased intra-individual reaction time variability. The pattern we obtained did not account for the observed slowing in reaction time. We suggest that this pattern reflects changes to spatial attention 
and early visual processing induced by sleep deprivation based on an application of the probe-period pattern to the stimulus phase. There was no evidence that memory scanning was related to the activation pattern based on a lack of change in expression of the pattern when applied to other set-size conditions. The activation pattern is also unlikely to be related to motor execution since it did not correlate with reaction time. Finally, there is insufficient evidence to accept or reject the notion that the activation pattern is related to binary decision.

\section{Acknowledgements}

This work was supported by the Defense Advanced Research Projects Agency (DARPA) grant DAAD 19-02-01-01147.

\section{References}

[1] G.E. Alexander, M.J. Mentis, J.D. Van Horn, C.L. Grady, K.F. Berman, M.L. Furey, P. Pietrini, S.I. Rapoport, M.B. Schapiro, J.R. Moeller, Individual differences in PET activation of object perception and attention systems predict face matching accuracy, NeuroReport 10 (1999) 1965-1971.

[2] H. Babkoff, T. Caspy, M. Mikulincer, Subjective sleepiness ratings: the effects of sleep deprivation, circadian rhythmicity and cognitive performance, Sleep 14 (1991) 534-539.

[3] H. Babkoff, T. Caspy, M. Mikulincer, H.C. Sing, Monotonic and rhythmic influences: a challenge for sleep deprivation research, Psychol. Bull. 109 (1991) 411-428.

[4] R.D. Badgaiyan, M.I. Posner, Mapping the cingulate cortex in response selection and monitoring, NeuroImage 7 (1998) 255-260.

[5] M.S. Beauchamp, L. Petit, T.M. Ellmore, J. Ingeholm, J.V. Haxby, A parametric fMRI study of overt and covert shifts of visuospatial attention, NeuroImage 14 (2001) 310-321.

[6] M.M. Botvinick, T.S. Braver, D.M. Barch, C.S. Carter, J.D. Cohen, Conflict monitoring and cognitive control, Psychol. Rev. 108 (2001) $624-652$.

[7] N. Burgess, E.A. Maguire, H.J. Spiers, J.A. O'Keefe, Temporoparietal and prefrontal network for retrieving the spatial context of lifelike events, NeuroImage 14 (2001) 439-453.

[8] V.D. Calhoun, T. Adali, V.B. McGinty, J.J. Pekar, T.D. Watson, G.D. Pearlson, fMRI activation in a visual-perception task: network of areas detected using the general linear model and independent components analysis, NeuroImage 14 (2001) 1080-1088.

[9] S. Carlson, S. Martinkauppi, P. Rama, E. Salli, A. Korvenoja, H.J. Aronen, Distribution of cortical activation during visuospatial $n$-back tasks as revealed by functional magnetic resonance imaging, Cereb. Cortex 8 (1998) 743-752.

[10] N. Cowan, The magical number 4 in short-term memory: a reconsideration of mental storage capacity, Behav. Brain Sci. 24 (2001) 87-114(discussion 114-85).

[11] M. D’Esposito, J.A. Detre, G.K. Aguirre, M. Stallcup, D.C. Alsop, L.J. Tippet, M.J. Farah, A functional MRI study of mental image generation, Neuropsychologia 35 (1997) 725-730.

[12] M. D'Esposito, B.R. Postle, B. Rypma, Prefrontal cortical contributions to working memory: evidence from event-related fMRI studies, Exp. Brain Res. 133 (2000) 3-11.

[13] D.F. Dinges, N.B. Kribbs, Performing while sleepy: effects of experimentally-induced sleepiness, in: T.H. Monk (Ed.), Sleep, Sleepiness and Performance, Wiley, New York, NY, 1991, pp. 97-128.
[14] S.P. Drummond, G.G. Brown, J.L. Stricker, R.B. Buxton, E.C. Wong, J.C. Gillin, Sleep deprivation-induced reduction in cortical functional response to serial subtraction, NeuroReport 10 (1999) 3745-3748.

[15] S.P. Drummond, G.G. Brown, J.C. Gillin, J.L. Stricker, E.C. Wong, R.B. Buxton, Altered brain response to verbal learning following sleep deprivation, Nature 403 (2000) 655-657.

[16] S.P. Drummond, J.C. Gillin, G.G. Brown, Increased cerebral response during a divided attention task following sleep deprivation, J. Sleep Res. 10 (2001) 85-92.

[17] B. Efron, R.J. Tibshirani, An Introduction to the Bootstrap, CRC Press, LLC, New York, 1994, 436 pp.

[18] I. Faillenot, H. Sakata, N. Costes, J. Decety, M. Jeannerod, Visual working memory for shape and 3D-orientation: a PET study, NeuroReport 8 (1997) 859-862.

[19] K.J. Friston, P. Fletcher, O. Josephs, A. Holmes, M.D. Rugg, R. Turner, Event-related fMRI: characterizing differential responses, NeuroImage 7 (1998) 30-40.

[20] K.J. Friston, O. Josephs, E. Zarahn, A.P. Holmes, S. Rouquette, J. Poline, To smooth or not to smooth? Bias and efficiency in fMRI time-series analysis, NeuroImage 12 (2000) 196-208.

[21] C.L. Grady, A.R. McIntosh, F. Bookstein, B. Horwitz, S.I. Rapoport, J.V. Haxby, Age-related changes in regional cerebral blood flow during working memory for faces, NeuroImage 8 (1998) 409-425.

[22] C.G. Habeck, Y. Stern, H.B. Posner, J.R. Moeller, Regional covariance analysis of event-related fMRI, SFN Abstracts (2002).

[23] C. Habeck, J. Hilton, E. Zarahn, J. Flynn, J.R. Moeller, Y. Stern, Relation of cognitive reserve and task performance to expression of regional covariance networks in an event-related fMRI study of nonverbal memory, NeuroImage 20 (2003) 1723-1733.

[24] C.G. Habeck, J.W. Krakauer, C. Ghez, Y. Stern, H.A. Sackeim, D. Eidelberg, J.R. Moeller, A new approach to spatial covariance modeling of functional brain imaging data: ordinal trend analysis, Neural Comput. (2003) (submitted for publication).

[25] Y. Harrison, J.A. Horne, The impact of sleep deprivation on decision making: a review, J. Exp. Psychol., Appl. 6 (2000) 236-249.

[26] J.A. Horne, Sleep loss and "divergent" thinking ability, Sleep 11 (1988) 528-536.

[27] J.A. Horne, Human sleep, sleep loss and behaviour. Implications for the prefrontal cortex and psychiatric disorder, Br. J. Psychiatry 162 (1993) 413-419.

[28] D.G. Humphrey, A.F. Kramer, R.R. Stanny, Influence of extended wakefulness on automatic and nonautomatic processing, Hum. Factors 36 1994, pp. 652-669.

[29] A.W. MacDonald III, J.D. Cohen, V.A. Stenger, C.S. Carter, Dissociating the role of the dorsolateral prefrontal and anterior cingulate cortex in cognitive control, Science 288 (2000) 1835-1838.

[30] M.E. McCarthy, W.F. Waters, Decreased attentional responsivity during sleep deprivation: orienting response latency, amplitude, and habituation, Sleep 20 (1997) 115-123.

[31] N.R. McFarland, S.N. Haber, Thalamic relay nuclei of the basal ganglia form both reciprocal and nonreciprocal cortical connections, linking multiple frontal cortical areas, J. Neurosci. 22 2002, pp. $8117-8132$.

[32] A.R. McIntosh, F.L. Bookstein, J.V. Haxby, C.L. Grady, Spatial pattern analysis of functional brain images using partial least squares, NeuroImage 3 (1996) 143-157.

[33] A.R. McIntosh, C.L. Grady, J.V. Haxby, L.G. Ungerleider, B. Horwitz, Changes in limbic and prefrontal functional interactions in a working memory task for faces, Cereb. Cortex 6 (1996) 571-584.

[34] V. Menon, C.D. White, S. Eliez, G.H. Glover, A.L. Reiss, Analysis of a distributed neural system involved in spatial information, novelty, and memory processing, Hum. Brain Mapp. 11 (2000) 117-129.

[35] T.H. Monk, J.E. Fookson, M.L. Moline, C.P. Pollak, Diurnal variation in mood and performance in a time-isolated environment, Chronobiol. Int. 2 (1985) 185-193.

[36] C. Moscovitch, S. Kapur, S. Kohler, S. Houle, Distinct neural correlates of visual long-term memory for spatial location and object iden- 
tity: a positron emission tomography study in humans, Proc. Natl. Acad. Sci. U. S. A. 92 (1995) 3721-3725.

[37] J.J. Pilcher, A.I. Huffcutt, Effects of sleep deprivation on performance: a meta-analysis, Sleep 19 (1996) 318-326.

[38] C.M. Portas, G. Rees, A.M. Howseman, O. Josephs, R. Turner, C.D Frith, A specific role for the thalamus in mediating the interaction of attention and arousal in humans, J. Neurosci. 18 (1998) 8979-8989.

[39] B.R. Postle, E. Zarahn, M. D'Esposito, Using event-related fMRI to assess delay-period activity during performance of spatial and nonspatial working memory tasks, Brain Res. Protoc. 5 (2000) 57-66.

[40] P. Rama, J.B. Sala, J.S. Gillen, J.J. Pekar, S.M. Courtney, Dissociation of the neural systems for working memory maintenance of verbal and nonspatial visual information, Cogn. Affect Behav. Neurosci. 1 (2001) $161-171$.

[41] B. Rypma, V. Prabhakaran, J.E. Desmond, G.H. Glover, J.D. Gabrieli, Load-dependent roles of frontal brain regions in the maintenance of working memory, NeuroImage 9 (1999) 216-226.

[42] B. Rypma, J.S. Berger, M. D’Esposito, The influence of workingmemory demand and subject performance on prefrontal cortical activity, J. Cogn. Neurosci. 14 (2002) 721-731.
[43] S. Sternberg, High-speed scanning in human memory, Science 153 (1966) 652-654

[44] S. Sternberg, Memory-scanning: mental processes revealed by reaction-time experiments, Am. Sci. 57 (1969) 421-457.

[45] M. Thomas, H. Sing, G. Belenky, H. Holcomb, H. Mayberg, R. Dannals, H. Wagner, D. Thorne, K. Popp, L. Rowland, A. Welsh, S. Balwinski, D. Redmond, Neural basis of alertness and cognitive performance impairments during sleepiness: I. Effects of $24 \mathrm{~h}$ of sleep deprivation on waking human regional brain activity, J. Sleep Res. 9 (2000) 335-352.

[46] K.J. Worsley, J.B. Poline, K.J. Friston, A.C. Evans, Characterizing the response of PET and fMRI data using multivariate linear models, NeuroImage 6 (1997) 305-319.

[47] J.C. Wu, J.C. Gillin, M.S. Buchsbaum, T. Hershey, E. Hazlett, N. Sicotte, W.E. Bunney Jr., The effect of sleep deprivation on cerebral glucose metabolic rate in normal humans assessed with positron emission tomography, Sleep 14 (1991) 155-162.

[48] E. Zarahn, Testing for neural responses during temporal components of trials with BOLD fMRI, NeuroImage 11 (2000) 783-796. 